\title{
microRNA-200a is an independent prognostic factor of hepatocellular carcinoma and induces cell cycle arrest by targeting CDK6
}

\author{
FENQIANG XIAO $^{1 *}$, WU ZHANG ${ }^{2 *}$, LIN ZHOU ${ }^{1}$, HAIYANG XIE $^{1}$, CHUNYANG XING $^{1}$, \\ SONGMING DING ${ }^{1}$, KANGJIE CHEN ${ }^{2}$ and SHUSEN ZHENG ${ }^{1,2}$ \\ ${ }^{1}$ Key Laboratory of Combined Multi-Organ Transplantation, Ministry of Public Health, Key Laboratory of \\ Organ Transplantation, Hangzhou, Zhejiang; ${ }^{2}$ Division of Hepatobiliary and Pancreatic Surgery, \\ First Affiliated Hospital, School of Medicine, Zhejiang University, Hangzhou, Zhejiang, P.R. China
}

Received May 23, 2013; Accepted August 16, 2013

DOI: 10.3892/or.2013.2715

\begin{abstract}
Deregulation of microRNA-200a (miR-200a) has been observed in different types of diseases, including cancers. However, the exact roles of miR-200a in hepatocellular carcinoma (HCC) are still largely unknown. We aimed to elucidate the prognostic implications of miR-200a and its biological function in HCC. Quantitative polymerase chain reaction was used to evaluate miR-200a expression. Western blotting was performed to evaluate the protein level. Gainof-function studies were performed to evaluate the roles of miR-200a in HCC. Our results revealed that miR-200a was frequently downregulated in HCC. In addition, multivariate analysis confirmed that miR-200a was significantly associated with the overall survival of HCC patients. In vitro assays demonstrated that miR-200a suppressed the proliferation of HCC cells by induction of G1 phase arrest. Furthermore, CDK6 was identified as a novel functional target of miR-200a. Our data indicate that miR-200a functions as a potential tumor suppressor in $\mathrm{HCC}$.
\end{abstract}

\section{Introduction}

Hepatocellular carcinoma (HCC) is one of the most common human malignancies and the leading cause of cancer-related mortality worldwide $(1,2)$. The development and progression of HCC involve the deregulation of genes that are crucial to cell cycle control, cell growth, apoptosis and cell migration. In the past few decades, studies have focused on investigating the

Correspondence to: Professor Shusen Zheng, First Affiliated Hospital, School of Medicine, Zhejiang University, 79\# Qingchun Road, Hangzhou, Zhejiang 310000, P.R. China

E-mail: shusenzheng@zju.edu.cn

${ }^{*}$ Contributed equally

Key words: hepatocellular carcinoma, microRNA-200a, CDK6, overall survival, cell cycle arrest protein-coding genes underlying the development and progression of HCC. Recently, reports suggest that misexpression of microRNAs (miRNAs) also contributes to tumorigenesis $(3,4)$.

miRNAs are small, endogenous, non-coding RNAs known to negatively regulate the expression of protein-coding genes $(5,6)$. miRNAs are involved in the regulation of various biological processes, such as development, cell proliferation, apoptosis and differentiation (7-9). Deregulation of miRNAs has been observed in various types of human cancer (10-13). Accumulating evidence indicates that miRNAs may function as oncogenes or tumor suppressor genes (TSGs) during tumor development and progression (4). Using an miRNA microarray, Murakami et al (14) determined that microRNA-200a (miR-200a) was significantly downregulated in HCC tissues when compared with the level in adjacent non-tumor tissues. However, the biological function of miR-200a in HCC and the detailed mechanisms in tumorigenesis are still largely unknown.

In the present study, we confirmed that downregulation of miR-200a occurred frequently in HCC tissues and cell lines. Additionally, miR-200a may be an independent prognostic factor. Moreover, ectopic expression of miR-200a dramatically inhibited cell proliferation and clonogenicity in vitro in HCC cells. Furthermore, gain-of-function studies revealed that miR-200a induced G1 phase arrest of HCC cells. Further study identified CDK6 as a novel functional target of miR-200a. Collectively, these findings indicate that miR-200a functions as a potential tumor suppressor in $\mathrm{HCC}$, providing a potential target for HCC therapy.

\section{Materials and methods}

Cell lines and tissue specimens. Immortalized normal liver cells (L02), human HCC cell lines (SK-HEP-1, HepG2, MHCC97H, MHCC97L, PLC, HuH7, SMMC-7721 and Bel-7402) and human embryonic kidney cells (HEK293T) were maintained in the recommended culture condition and incubated at $37^{\circ} \mathrm{C}$ in a humidified environment containing $5 \%$ $\mathrm{CO}_{2}$. Paired $\mathrm{HCC}$ and adjacent non-tumor liver tissues were collected from patients undergoing liver transplantation or 
Table I. Association of miR-200a expression with clinicopathological parameters of the HCC cases.

\begin{tabular}{|c|c|c|c|}
\hline Factors & $\begin{array}{l}\text { Low expression } \\
\text { group, } \mathrm{n}(\%) \\
(\mathrm{n}=64)\end{array}$ & $\begin{array}{l}\text { High expression } \\
\text { group, } \mathrm{n}(\%) \\
(\mathrm{n}=56)\end{array}$ & P-value ${ }^{a}$ \\
\hline Age (years) & & & 0.824 \\
\hline$\leq 50$ & $34(53.1)$ & $30(53.6)$ & \\
\hline$>50$ & $30(46.9)$ & $26(46.4)$ & \\
\hline Gender & & & 0.578 \\
\hline Male & 49 (76.6) & $40(71.4)$ & \\
\hline Female & $15(23.4)$ & $16(28.6)$ & \\
\hline $\mathrm{HBV}$ & & & 0.653 \\
\hline Positive & $52(81.3)$ & $45(80.4)$ & \\
\hline Negative & $12(18.7)$ & $11(19.6)$ & \\
\hline Cirrhosis & & & 0.579 \\
\hline Positive & $51(79.7)$ & $42(75.0)$ & \\
\hline Negative & $13(20.3)$ & $14(25.0)$ & \\
\hline Tumor size $(\mathrm{cm})$ & & & 0.861 \\
\hline$\leq 5$ & $42(65.6)$ & $37(66.1)$ & \\
\hline$>5$ & $22(34.4)$ & $19(33.9)$ & \\
\hline Tumor number & & & 0.924 \\
\hline Single & $41(64.1)$ & $36(64.3)$ & \\
\hline Multiple & $23(35.9)$ & $21(35.7)$ & \\
\hline Histologic grade & & & 0.015 \\
\hline Well and moderate & $17(26.6)$ & $26(50.0)$ & \\
\hline Poor and others & $47(73.4)$ & $26(50.0)$ & \\
\hline TNM stage & & & 0.012 \\
\hline I & $18(28.1)$ & $34(60.7)$ & \\
\hline II and III & $46(71.9)$ & $22(39.3)$ & \\
\hline $\mathrm{AFP}(\mathrm{ng} / \mathrm{ml})$ & & & 0.025 \\
\hline$\leq 400$ & $26(40.6)$ & $33(58.9)$ & \\
\hline$>400$ & $38(59.4)$ & $23(41.1)$ & \\
\hline PVTT & & & 0.023 \\
\hline Positive & $24(37.5)$ & $13(23.2)$ & \\
\hline Negative & $40(62.5)$ & $43(76.8)$ & \\
\hline
\end{tabular}

${ }^{a}$ Chi-square test (Pearson's Chi-square). TNM, tumor-node-metastasis; AFP, $\alpha$-fetoprotein; PVTT, portal vein tumor thrombi.

partial hepatectomy at The First Affiliated Hospital, School of Medicine, Zhejiang University (Hangzhou, Zhejiang, China). Written informed consent was obtained from each patient. A total of 120 patients had a clear histologic diagnosis of HCC with complete clinicopathological data, and all patients were followed up for survival analysis. None of the patients received radiotherapy or chemotherapy before surgery. All sample data were obtained from clinical and pathologic records and are summarized in Table I.

Oligoribonucleotides. miR-200a mimic and the control RNA duplex (referred to as NC) were used for the transient gain-of-function study. The small interfering RNA (siRNA) targeting the human CDK6 transcript was designated as siCDK6. The NC for both the miRNA mimic and siRNA was non-homologous to any human genome sequence. All the RNA oligoribonucleotides (Table II) were purchased from Genepharma (Shanghai, China). All oligoribonucleotides used in the present study are shown in Table II.

RNA extraction and quantitative reverse transcriptase-polymerase chain reaction ( $q R T-P C R)$. Total RNA from cell lines and clinical samples was isolated using the mirVana miRNA Isolation kit (Ambion). qRT-PCR was performed to detect the expression of miR-200a in various cell lines and clinical samples and the expression of CDK6 at the mRNA level. RNA was reverse transcribed using One Step PrimeScript miRNA cDNA Synthesis kit (Takara, Japan). The cDNA was then quantified by real-time RT-PCR using SYBR Premix Ex Taq (Takara). All PCR reactions were performed using the ABI7500 system (Applied Biosystems, Foster City, CA, USA). U6 or glyceraldehyde 3-phosphate dehydrogenase (GAPDH) was used as an internal control. All primers used are listed in Table II.

Western blotting. Western blotting was performed to detect the gene expression at the protein level. Protein was extracted from transfected SK-HEP-1 cells using modified RIPA buffer in the presence of proteinase inhibitor cocktail. Equivalent quantities $(30-50 \mu \mathrm{g})$ of protein were separated on $10 \%$ SDS-polyacrylamide gels and transferred to polyvinylidene difluoride membranes. Membranes were blocked with 5\% non-fat milk and then incubated overnight at $4^{\circ} \mathrm{C}$ with the appropriate primary antibody at the dilutions specified by the manufacturer. The membranes were then washed three times in $10 \mathrm{ml}$ TBST and incubated with the corresponding horseradish peroxidase (HRP)-conjugated secondary antibody at a dilution of 1:2,000 for $1 \mathrm{~h}$. The bound secondary antibody was detected using an Enhanced chemiluminescence (ECL) system (Pierce Biotechnology, Inc., Rockford, IL, USA). Primary antibodies were as follows: anti-CDK6, anti-cyclin E2, anti-Rb, anti-phospho-Ser780-Rb, anti-cdc2, (Cell Signaling Technology), anti-phospho-Ser795-Rb and anti- $\beta$-actin (Epitomics).

Cell transfection. All transfections were performed using Lipofectamine ${ }^{\circledR} 2000$ (Invitrogen) according to the manufacturer's instructions. Briefly, SK-HEP-1, Huh7 or HEK293T cells were transfected with DNA, miRNA mimic, siRNA or NC. All RNA transfections were performed at a final concentration of $50 \mathrm{nM}$. Cells were collected for assay $48 \mathrm{~h}$ after transfection.

Cell proliferation assay. SK-HEP-1 and Huh7 cells seeded at a density of 4,000/well into 96-well plates were transfected with miR-200a mimic or NC. After incubating the cells for the specified time (1, 2, 3 or 4 days), a cell proliferation assay was performed using Cell Counting Kit-8 (CCK-8) (Dojindo) according to the manufacturer's instructions. The solution absorbance was measured spectrophotometrically at $450 \mathrm{~nm}$ with the MRX II absorbance reader (Dynex Technologies, Chantilly, VA, USA). The experiments were performed in triplicate.

Colony formation assay. Aliquots of viable transfected SK-HEP-1 and Huh7 cells (1,000/well) were placed in 6-well 
Table II. List of the oligonucleotides used in the present study.

\begin{tabular}{|c|c|}
\hline Name & Sequence $\left(5^{\prime} \rightarrow 3^{\prime}\right)^{\mathrm{a}}$ \\
\hline \multicolumn{2}{|l|}{ miRNA and siRNA duplexes } \\
\hline miR-200a mimic (sense) & UAACACUGUCUGGUAACGAUGU \\
\hline $\mathrm{NC}$ (sense) & ACUACUGAGUGACAGUAGA[dT][dT] \\
\hline siCDK6 (sense) & CGGACAGTAUUAGAUACAUTT \\
\hline \multicolumn{2}{|l|}{ Primers for RT-PCR } \\
\hline miR-200a, F & CTCTAACACTGTCTGGTAACGATGT \\
\hline CDK6, F & CAGCAGCGGACAAATAAA \\
\hline CDK6, R & CTGGGAGTCCAATCACGT \\
\hline GAPDH, F & AAGGTGAAGGTCGGAGTCA \\
\hline GAPDH, R & GGAAGATGGTGATGGGATTT \\
\hline \multicolumn{2}{|l|}{ Primers for 3'UTR cloning } \\
\hline CDK6-utr, F & CGGAGCTCTTTCTAACCTTGAATGCTGC \\
\hline CDK6-utr, R & CGCGTCGACAGAAAAGAAATGCTGAGGAC \\
\hline CDK6-mut, F & $\begin{array}{l}\text { GTGTGTGTGTGTGTGTATGTGAGAGATTCTGTGATCTTTTAA } \\
\text { TCACAAACTTTTTGTAAACGACAAGAATAATTCAATTTTAAA }\end{array}$ \\
\hline CDK6-mut, R & $\begin{array}{l}\text { TTTAAAATTGAATTATTCTTGTCGTTTACAAAAAGTTTGTGATT } \\
\text { AAAAGATCACAGAATCTCTCACATACACACACACACACAC }\end{array}$ \\
\hline
\end{tabular}

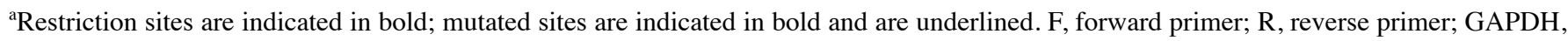
glyceraldehyde 3-phosphate dehydrogenase.

plates $24 \mathrm{~h}$ after transfection and maintained in complete medium for 2 weeks. Colony formation was evaluated by staining the cells with $0.1 \%$ crystal violet. The rate of colony formation was calculated using the following equation: Colony formation rate $=$ (number of colonies/number of seeded cells) x $100 \%$. The experiments were performed in triplicate.

Cell cycle analysis. Forty-eight hours after transfection, SK-HEP-1 and Huh7 cells were harvested, washed with PBS and fixed in $70 \%$ ethanol at $4^{\circ} \mathrm{C}$ overnight. Staining for DNA content was performed using a DNA Prep Stain (Beckman Coulter, Fullerton, CA, USA). Cell populations in the G0/G1, $\mathrm{S}$ and $\mathrm{G} 2 / \mathrm{M}$ phases were determined using the BD ${ }^{\mathrm{TM}}$ LSRII flow cytometry system with FACSDiva software (both from BD Bioscience, Franklin Lakes, USA). Data were analyzed using ModFit LT software. The experiments were performed in triplicate.

Target prediction. To predict the target genes and the 3' UTR binding sites bound by the seed region of miR-200a, the TargetScan (15) (http://www.targetscan.org/) and PicTar (16) (http://pictar.mdc-berlin.de/) databases were used. CDK6 was chosen as the target candidate according to both TargetScan and PicTar databases.

Vector construction and luciferase reporter assay. The 3' UTR of CDK6 was amplified by PCR. The amplified sequence was subcloned and ligated into the pmirGLO Dual-Luciferase miRNA Target Expression Vector (Promega, Madison, WI, USA). The recombinant reporter vector was identified by sequencing and termed the wild-type (Wt). To create the miR-200a binding site mutants, the binding region of the seed sequence (5'-AGTGTT-3') was mutated to the sequence 5'-TCACAA-3', using the QuikChange Lightning Site-Directed Mutagenesis kit (Stratagene) according to the manufacturer's protocol. The recombinant vector was confirmed by sequencing and termed the mutant type (Mut). $293 \mathrm{~T}$ cells plated in a 24-well plate were co-transfected with $50 \mathrm{nM}$ of either the miR-200a mimic or NC and $100 \mathrm{ng}$ of pmirGLO vector comprising Wt or Mut 3' UTR of CDK6 using Lipofectamine ${ }^{\circledR} 2000$. Forty-eight hours after transfection, the relative luciferase activity was measured by the DualLuciferase Reporter Assay System (Promega). All transfection experiments were performed in triplicate.

Statistical analysis. Data are presented as the means \pm SD. Comparisons were made by using the Student's t-test, the $\chi^{2}$ test and the log-rank test. Overall survival rates were calculated actuarially according to the Kaplan-Meier method. Variables with a value of $\mathrm{P}<0.05$ in univariate analysis were used in a subsequent multivariate analysis based on the Cox proportional hazards model. A value of $\mathrm{P}<0.05$ was considered to indicate a statistically significant result.

\section{Results}

miR-200a is frequently downregulated in HCC cell lines and tissues. To further confirm the expression pattern of miR-200a in HCC, we detected the expression of miR-200a in 120 paired HCC and adjacent non-cancerous liver tissues by real-time qRT-PCR. Compared with the adjacent non-tumor tissues, significant downregulation of miR-200a was observed in the HCC tissues (Fig. 1A). Furthermore, the expression of miR-200a was noticeably reduced in 6 of $8(75.0 \%)$ HCC cell 

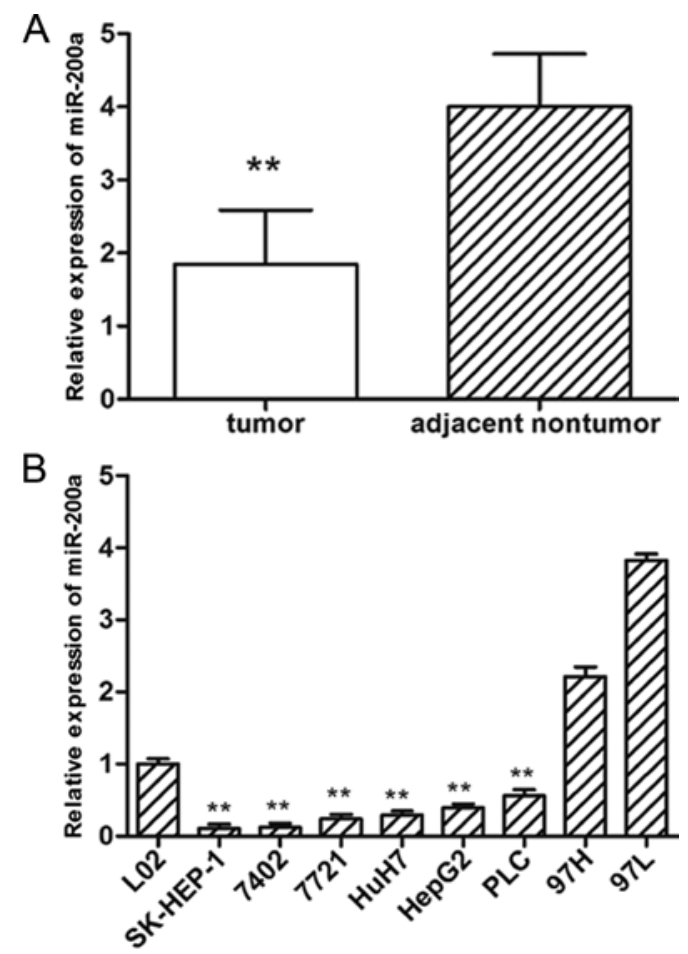

Figure 1. Expression pattern of miR-200a in hepatocellular carcinoma (HCC) cell lines and tissues. (A) A lower expression level of miR-200a was detected in tumors when compared with expression in the adjacent non-tumorous tissues in 120 HCC patients. (B) Relative expression levels of miR-200a in the indicated HCC cell lines $\left({ }^{*} \mathrm{P}<0.05 ;{ }^{* *} \mathrm{P}<0.01 ;{ }^{* * *} \mathrm{P}<0.001\right)$.

lines examined when compared with the expression in the normal liver cell line (L02) (Fig. 1B). These results suggest that reduced miR-200a expression is a frequent event in human HCC and may be involved in tumorigenesis.

Downregulation of miR-200a is associated with worse prognosis. In the present study, patients with expression values less than the average level of miR-200a in HCC tissues (1.87, normalized to U6) were assigned to the low expression group $(n=64)$ whereas patients with values above the average were assigned to the high expression group $(\mathrm{n}=56)$. Our data showed a significant association between miR-200a expression and the AFP level, histologic grade, TNM stage and PVTT (Table I). Furthermore, the Kaplan-Meier method revealed that a lower miR-200a level was associated with shorter overall survival $(\mathrm{P}<0.01)$ (Fig. 2). Multivariate analysis further confirmed that a reduced miR-200a level was an independent and significant prognostic factor for survival (HR, 2.621; CI, 1.116-4.642; $\mathrm{P}=0.004$; Table III). Collectively, these data suggest that deregulation of miR-200a may contribute to the development and progression of HCC.

Ectopic expression of miR-200a significantly inhibits cell proliferation. The frequent downregulation of miR-200a in HCC cell lines and tissues suggests that miR-200a may serve as a potential TSG in HCC. To test the potential tumor-suppressor role of miR-200a in HCC, the effect of ectopic miR-200a on cell growth was evaluated using the CCK- 8 cell viability assay. The expression of miR-200a was markedly increased in the SK-HEP-1 and Huh7 cells transfected with $50 \mathrm{nM}$
Table III. Univariate and multivariate analysis for overall survival of the HCC patients.

\begin{tabular}{lcc}
\hline Clinical variables & HR $(95 \% \mathrm{CI})$ & P-value \\
\hline Univariate analysis & & \\
Age & $1.021(0.726-1.346)$ & 0.786 \\
Gender & $0.953(0.568-1.775)$ & 0.653 \\
HBV & $1.157(0.563-2.195)$ & 0.874 \\
Cirrhosis & $1.652(0.838-3.688)$ & 0.257 \\
Tumor size & $1.568(1.056-2.446)$ & 0.077 \\
Tumor no. & $2.451(1.547-4.639)$ & 0.276 \\
TNM stage & $2.774(1.674-4.797)$ & $\mathbf{0 . 0 1 2}$ \\
Histologic grade & $1.826(1.113-2.893)$ & $\mathbf{0 . 0 2 4}$ \\
PVTT & $3.639(2.538-7.531)$ & $\mathbf{0 . 0 1 6}$ \\
AFP & $2.635(1.368-5.062)$ & $\mathbf{0 . 0 1 7}$ \\
miR-200a expression & $2.845(1.876-4.874)$ & $\mathbf{0 . 0 0 2}$ \\
Multivariate analysis & & \\
TNM stage & $2.053(1.586-4.753)$ & $\mathbf{0 . 0 1 2}$ \\
Histologic grade & $1.431(0.690-2.968)$ & 0.336 \\
PVTT & $0.649(0.642-1.276)$ & 0.172 \\
AFP & $1.153(0.645-2.297)$ & 0.664 \\
miR-200a expression & $2.621(1.116-4.642)$ & $\mathbf{0 . 0 0 4}$ \\
\hline
\end{tabular}

Analysis was conducted on $120 \mathrm{HCC}$ cases. Hazard ratios (95\% CIs) and $\mathrm{P}$-values were calculated using univariate or multivariate Cox proportional hazards regression. $\mathrm{P}$-values in bold indicate statistical significance. HR, hazard ratio; CI, confidence interval; TNM, tumor-node-metastasis; PVTT, portal vein tumor thrombi; AFP, $\alpha$-fetoprotein.

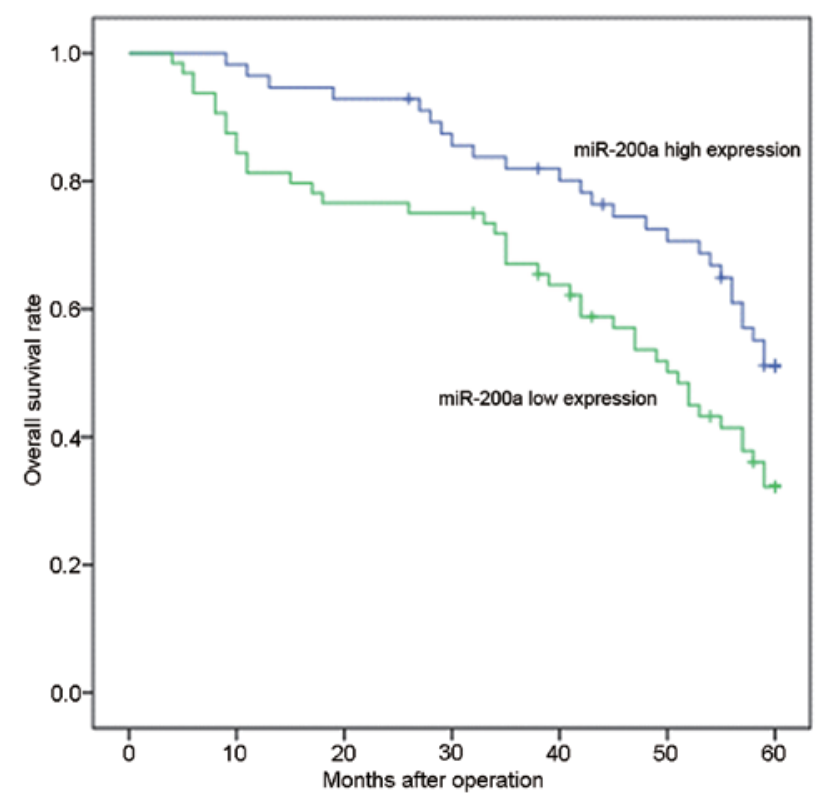

Figure 2. Kaplan-Meier overall survival curves for the hepatocellular carcinoma patients according to the level of miR-200a expression. Patients in the low miR-200a expression group $(n=64)$ had a significantly shorter overall survival than those in the high miR-200a expression group $(\mathrm{n}=56)(\mathrm{P}<0.01)$.

miR-200a mimic when compared with NC, normalized to U6 (Fig. 3A). Our data revealed that miR-200a overexpression 
A

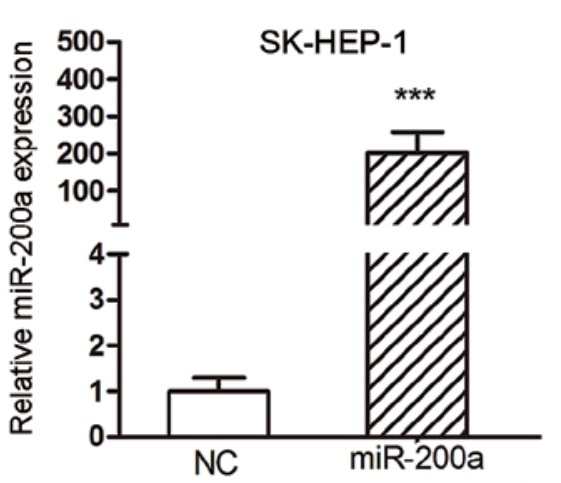

B

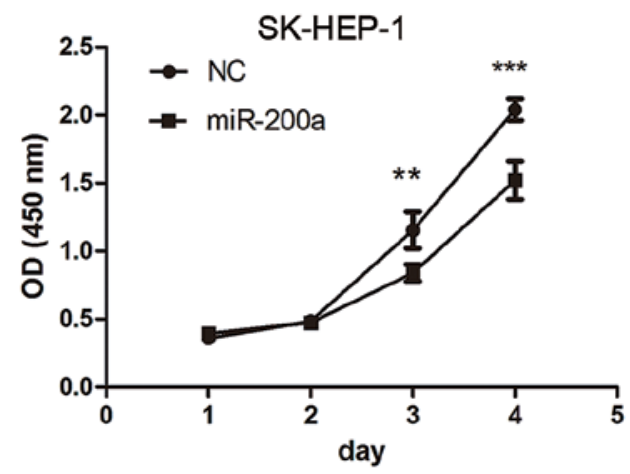

C

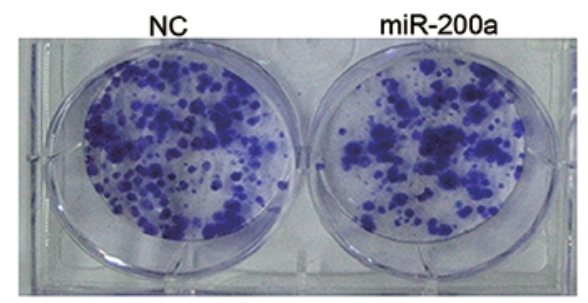

SK-HEP-1

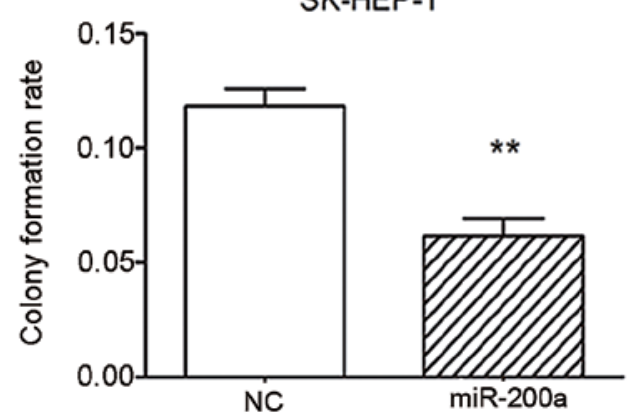

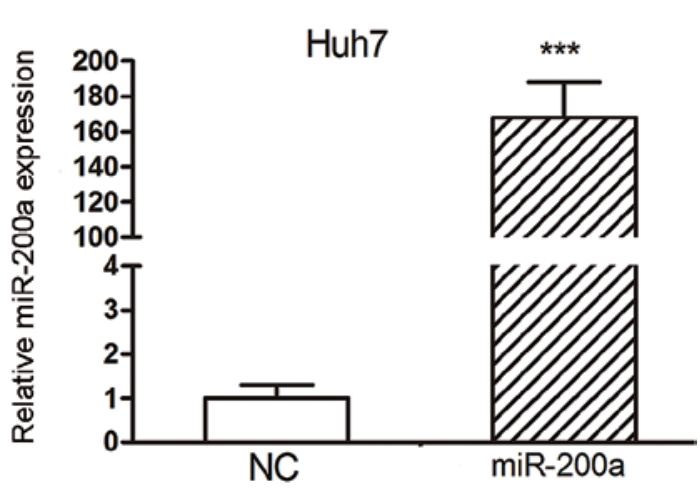

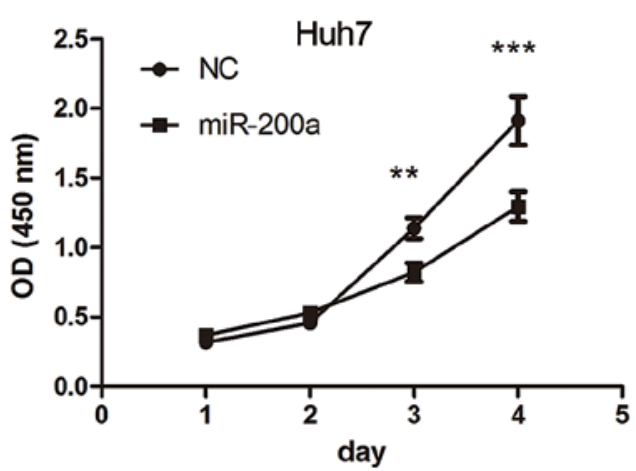

NC

miR-200a

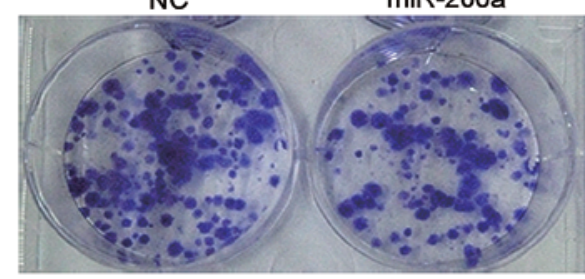

Huh7

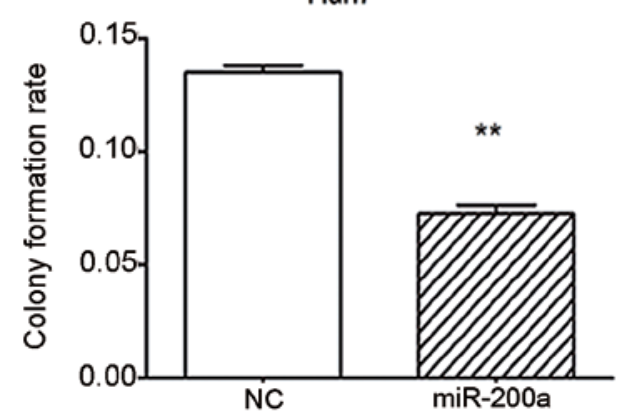

Figure 3. Effect of miR-200a on cell proliferation and clonogenicity in vitro. (A) Expression of miR-200a in SK-HEP-1 and Huh7 cells following transfection with the miR-200a mimic or NC duplex. Expression of miR-200a was normalized to U6. (B) Effects of miR-200a overexpression on the proliferation of SK-HEP-1 and Huh7 cells were determined by CCK-8 assay. (C) The colony formation rate was significantly reduced in the miR-200a-treated group when compared with the rate in the NC-treated group for both SK-HEP-1 and Huh7 cells. ${ }^{* * *} \mathrm{P}<0.01 ;{ }^{* * * *} \mathrm{P}<0.001$.

significantly inhibited cell proliferation in SK-HEP-1 and Huh7 cells compared to NC. At day 1, the OD values of the miR-200a mimic and NC control cells were not significantly different. However, from day 3 onwards, the OD values of the miR-200a-treated SK-HEP-1 and Huh7 cells were significantly lower than the value of the control cells (Fig. 3B). These data indicated that miR-200a inhibited cell proliferation in vitro.

miR-200a suppresses clonogenicity in vitro. To further investigate the potential role of miR-200a in tumorigenesis, a colony formation assay was performed in SK-HEP-1 and Huh7 cells. Cells were transfected with the miR-200a mimic or NC duplex, and then allowed to grow to a very low density. Notably, SK-HEP-1 and Huh7 cells transfected with $50 \mathrm{nM}$ miR-200a mimic displayed much fewer and smaller colonies compared with the NC duplex-transfected cells (Fig. 3C), suggesting a growth-inhibitory role of miR-200a.

Ectopic expression of miR-200a induces cell cycle arrest. To explore the mechanisms underlying the suppression of tumor growth by miR-200a, the effect of miR-200a on cell cycle progression was investigated by flow cytometry. The assay 
A

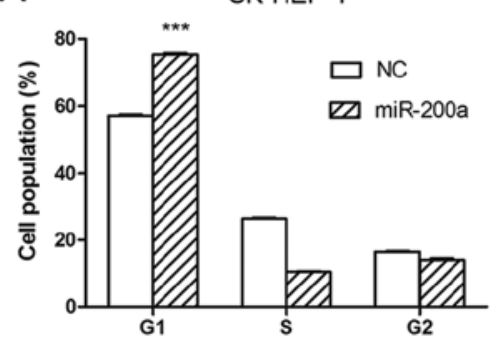

B

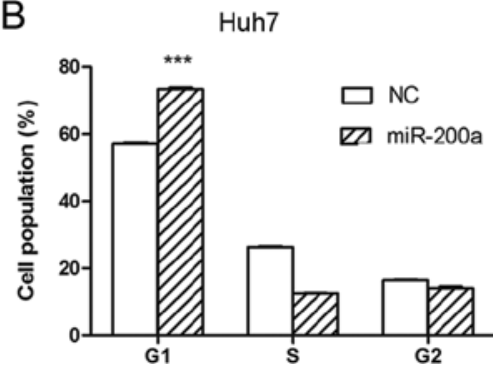

C

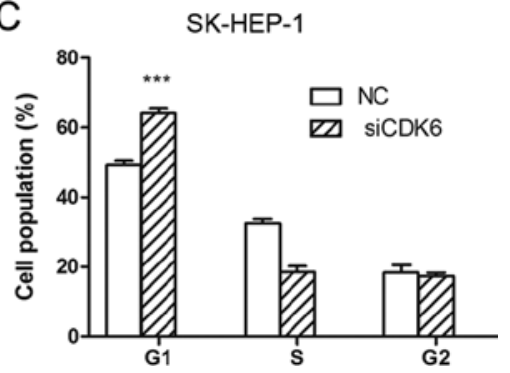

Figure 4. Cell cycle distribution of cells at $48 \mathrm{~h}$ following treatment with miR-200a mimic or siCDK6. Forty-eight hours after transfection, cells were subjected to flow cytometry. (A and B) Overexpression of miR-200a induced a significant G1-phase arrest of SK-HEP-1 and Huh7 cells. (C) Knockdown of CDK6 induced a significant accumulation of G1-phase cells and blocked G1/S transition in SK-HEP-1 cells . ${ }^{* * *} \mathrm{P}<0.001$.

A

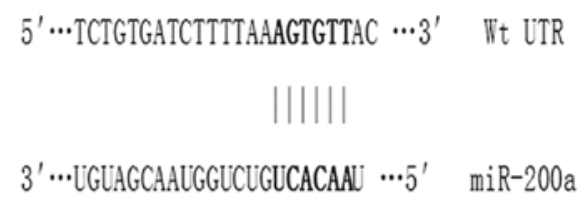

$5^{\prime} \cdots$ TCTGTGATCTTTTAATCACAAC $\cdots 3^{\prime}$ Nut UTR

B

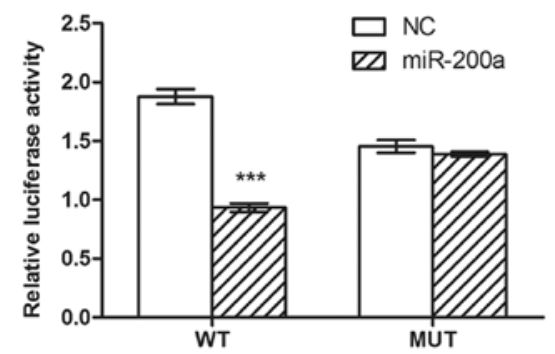

C
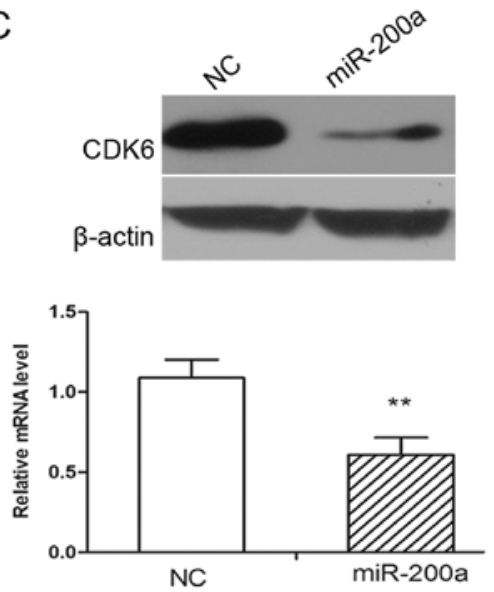

D

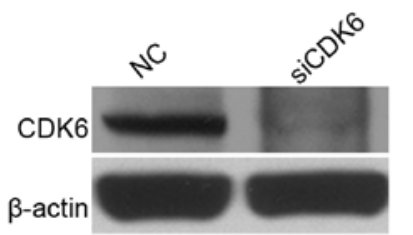

Figure 5. CDK6 is a direct target of miR-200a. (A) The putative miR-200a binding sequence in the 3' UTR of CDK6 mRNA. Mutation was generated on the CDK6 3' UTR sequence in the complementary site for the seed region of miR-200a. (B) Analysis of luciferase activity. 293T cells were cotransfected with $50 \mathrm{nM}$ of either miR-200a or NC and $100 \mathrm{ng}$ pmirGLO vector containing wild-type (Wt) or mutant type (Mut) 3 ' UTR of CDK6 (indicated as WT or MUT on the X-axis). The relative firefly luciferase activity normalized with Renilla luciferase was measured $48 \mathrm{~h}$ after transfection. (C) Effect of miR-200a on the expression of endogenous CDK6 at the protein and mRNA levels. (D) Effect of siCDK6 on the expression of endogenous CDK6 at the protein level. ${ }^{* *} \mathrm{P}<0.01$; ${ }^{* * *} \mathrm{P}<0.001$

showed that overexpression of miR-200a resulted in an accumulation of a G1 population in the SK-HEP-1 (Fig. 4A) and Huh7 cells (Fig. 4B). These results indicated that miR-200a inhibited HCC cell proliferation by inducing cell cycle arrest at the G1 phase.

CDK6 is a direct functional target of miR-200a. It is generally believed that miRNAs exert their function through negatively regulating the expression of their downstream target genes. To further elucidate the molecular mechanism by which miR-200a inhibits G1/S transition, CDK6 was predicted as a potential target of miR-200a by TargetScan and PicTar. A single putative miR-200a-binding site was mapped in the 3' UTR of CDK6 (Fig. 5A). To validate whether CDK6 is a direct target of miR-200a, a human CDK6 3' UTR fragment containing Wt or the mutant (Mut) miR-200a binding sequence
(Fig. 5A) was cloned downstream of the firefly luciferase reporter gene in pmirGLO. In HEK293 cells cotransfected with the reporter plasmids and the miR-200a mimic or NC duplex, the luciferase activity of the reporter that contained Wt 3' UTR was significantly suppressed by miR-200a mimic, but the luciferase activity of the mutant reporter was unaffected (Fig. 5B), indicating that miR-200a may suppress gene expression through miR-200a binding sequence at the $3^{\prime}$ UTR of CDK6. Additionally, transfection of the miR-200a mimic decreased CDK6 expression in SK-HEP-1 cells at both the protein and mRNA levels (Fig. 5C), suggesting that CDK6 expression is inhibited by $\mathrm{miR}-200 \mathrm{a}$ at the post-transcriptional level. Collectively, the results showed that miR-200a regulated the expression of endogenous CDK6 by directly targeting the 3' UTR of CDK6 mRNA and that CDK6 is a novel target of miR-200a. 


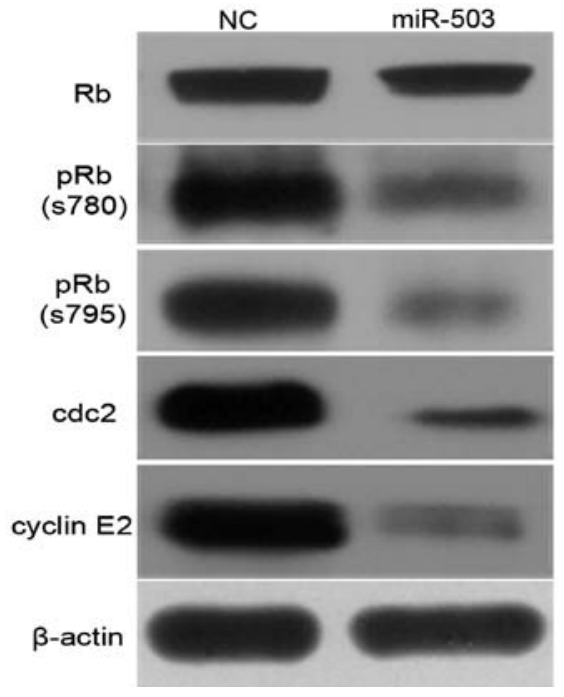

Figure 6. Effect of ectopic expression of miR-200a on retinoblastoma $(\mathrm{Rb})$ phosphorylation status and the downstream genes of E2F3. miR-200a overexpression significantly inhibited the phosphorylation of Rb on Ser780 and Ser795 and the expression of cdc2 and cyclin E2. Gene expression was examined by western blotting. $\mathrm{Rb}$, total $\mathrm{Rb}$ protein; $\mathrm{pRb}$, phosphorylated $\mathrm{Rb}$. $\beta$-actin, internal control.

miR-200a is involved in G1/S transition through the downregulation of $C D K 6$. To explore the role of CDK6 in the miR-200a-regulated G1/S transition, we investigated whether knockdown of CDK6 may phenocopy the effect of miR-200a overexpression. SK-HEP-1 cells were transfected with siRNA duplex targeting $C D K 6$, which resulted in a significant reduction in the protein levels (Fig. 5D). The silencing of CDK6 led to G1-phase arrest (Fig. 4C), phenocopying the outcome of miR-200a overexpression.

CDK6 is one of the crucial molecules that initiate the phosphorylation of retinoblastoma $(\mathrm{Rb})$, which results in the release of $\mathrm{E} 2 \mathrm{~F}$ and subsequently the transactivation of genes required for S-phase entry. Therefore, we investigated whether miR-200a could attenuate these events. Ectopic expression of miR-200a caused a reduction in phosphorylated $\mathrm{Rb}$ protein, however little effect on the total Rb protein was noted (Fig. 6). Moreover, the effect of miR-200a on endogenous genes such as cdc2 and cyclin E2, downstream genes of E2F3, was further investigated. Overexpression of miR-200a induced significant downregulation of cdc2 and cyclin E2 in SK-HEP-1 cells (Fig. 6).

These data indicate that miR-200a may induce cell cycle arrest at G1 phase by directly inhibiting CDK6 through the $\mathrm{Rb}-\mathrm{E} 2 \mathrm{~F}$ signaling pathway.

\section{Discussion}

Although deregulation of miRNAs has been observed in various types of cancers $(11,17)$, the molecular mechanisms by which miRNAs regulate the process of carcinogenesis and the biological behavior of cancer cells are still largely unknown. Growing evidence has revealed that a defect in cell cycle control is an essential step during carcinogenesis $(18,19)$. miR-138 was found to induce cell cycle arrest by targeting cyclin D3 in HCC (20). miR-195 suppressed G1/S transition of human HCC cells by targeting cyclin D1, CDK6 and E2F3 (21). Therefore, misexpression of cell cycle-related miRNAs may facilitate tumorigenesis. A previous miRNA microarray revealed that miR-200a was significantly downregulated in HCC (14). Recently altered expression of miR-200a has been observed in different types of diseases, including cancers. Downregulation of the miR-200 family expression was recently shown to be associated with tumor progression (22). Epithelial-to-mesenchymal transition (EMT), a crucial process in the transformation of tumor cells into aggressive metastatic cancer cells, also seems to be tightly regulated by this family $(23,24)$. Saydam et al $(25)$ reported that downregulation of miR-200a in brain tumors promoted tumor growth by upregulating cyclin $\mathrm{D} 1$ and $\beta$-catenin in vitro and in vivo. Similarly, it was reported that the overexpression of miR-200a inhibited nasopharyngeal carcinoma cell growth, migration and invasion by downregulation of $\beta$-catenin and Smad interacting protein 1 (26).

Little is known concerning the role of the miR-200 family in cell growth and proliferation in HCC. Hung et al (27) found that members of the miR-200 family (miR-200a and miR-200b) were involved in HCC migration by regulating E-cadherin expression. However, the role of miR-200 family members and the relative molecular mechanism in HCC remain largely unclear. In the present study, we attempted to elucidate the role of miR-200a in HCC. Our findings revealed that miR-200a was frequently downregulated in human HCC tissues. Lee et al (28) previously reported that the miR-200 family, including miR-200a, was upregulated in endometrial endometrioid carcinomas when compared with expression in normal endometrial tissues. This discrepancy may be due to the use of different cell lines. In the present study, multivariate analysis revealed that miR-200a is an independent prognostic factor for survival. In addition, gain-of-function studies indicated that overexpression of miR-200a suppressed cell proliferation by induction of cell cycle arrest in HCC cell lines. Moreover, we identified CDK6 as a direct target of miR-200a in HCC, which may provide new insights into the mechanisms underlying tumorigenesis. These results imply the important role of miR-200a in the tumorigenesis of HCC.

The family of CDKs and their activating partners (cyclins) are crucial molecules involved in cell cycle control. The G1/S transition is regulated primarily by D-type cyclins in complex with CDK4/CDK6 and E-type cyclins in complex with CDK2. These complexes cooperate in phosphorylating and preventing $\mathrm{Rb}$ binding to $\mathrm{E} 2 \mathrm{~F}$, thus activating E2F-mediated transcription and driving cells from G1 into $\mathrm{S}$ phase (29). However, the detailed mechanisms of cell cycle regulation in $\mathrm{HCC}$ require further investigation. Our results showed that miR-200a induced cell cycle arrest of HCC cells by downregulation of CDK6 at both the mRNA and protein levels. Further investigation revealed that ectopic miR-200a expression significantly inhibited $\mathrm{Rb}$ phosphorylation and the expression level of cdc2 and cyclin E2 at the protein level (Fig. 6). Collectively our results indicate that miR-200a may be involved in the induction of cell cycle arrest in HCC through the $\mathrm{CDK} / \mathrm{Rb} / \mathrm{E} 2 \mathrm{~F}$ pathway.

The underlying mechanism responsible for the decreased expression of miR-200a in HCC remains unknown. miR-200a is located on chromosome $1 \mathrm{p} 36$, a region which is often deleted in several types of cancer $(30,31)$, providing a plausible 
mechanism for the reduced miR-200a expression in HCC. In addition, promoter hypermethylation or transcriptional regulation may account at least in part for the reduced miR-200a expression in HCC. Further investigation is required to verify this hypothesis.

In summary, we confirmed the frequent downregulation of miR-200a in HCC and revealed the potential role of miR-200a in tumorigenesis. Our data indicate that miR-200a may function as a potential tumor suppressor and could be an independent prognostic marker in HCC patients. Therefore, miR-200a may serve as a useful therapeutic target for HCC therapy.

\section{Acknowledgements}

We thank Jilei Fu for the collection of clinical data and Rong Su for her excellent technical assistance. The present study was supported by the following grants and foundations: National S\&T Major Project (2012zx10002-017); NSFC for Innovative Research Group (81121002); the National Natural Science Foundation of China (81201944); Zhejiang Provincial Natural Science Foundation of China for Distinguished Young Scholars (no. R2110125).

\section{References}

1. Parkin DM, Bray F, Ferlay J and Pisani P: Global cancer statistics, 2002. CA Cancer J Clin 55: 74-108, 2005.

2. Aravalli RN, Steer CJ and Cressman EN: Molecular mechanisms of hepatocellular carcinoma. Hepatology 48: 2047-2063, 2008.

3. Calin GA and Croce CM: MicroRNA signatures in human cancers. Nat Rev Cancer 6: 857-866, 2006.

4. Esquela-Kerscher A and Slack FJ: Oncomirs - microRNAs with a role in cancer. Nat Rev Cancer 6: 259-269, 2006.

5. Ambros V: The functions of animal microRNAs. Nature 431: 350-355, 2004.

6. Bartel DP: MicroRNAs: genomics, biogenesis, mechanism, and function. Cell 116: 281-297, 2004.

7. Schratt GM, Tuebing F, Nigh EA, et al: A brain-specific microRNA regulates dendritic spine development. Nature 439: 283-289, 2006.

8. Brennecke J, Hipfner DR, Stark A, Russell RB and Cohen SM: bantam encodes a developmentally regulated microRNA that controls cell proliferation and regulates the proapoptotic gene hid in Drosophila. Cell 113: 25-36, 2003.

9. Bartel DP: MicroRNAs: target recognition and regulatory functions. Cell 136: 215-233, 2009.

10. Su H, Yang JR, Xu T, et al: MicroRNA-101, down-regulated in hepatocellular carcinoma, promotes apoptosis and suppresses tumorigenicity. Cancer Res 69: 1135-1142, 2009.

11. Lu J, Getz G, Miska EA, et al: MicroRNA expression profiles classify human cancers. Nature 435: 834-838, 2005.

12. Yanaihara N, Caplen N, Bowman E, et al: Unique microRNA molecular profiles in lung cancer diagnosis and prognosis. Cancer Cell 9: 189-198, 2006.
13. Iorio MV, Visone R, Di Leva G, et al: MicroRNA signatures in human ovarian cancer. Cancer Res 67: 8699-8707, 2007.

14. Murakami Y, Yasuda T, Saigo K, et al: Comprehensive analysis of microRNA expression patterns in hepatocellular carcinoma and non-tumorous tissues. Oncogene 25: 2537-2545, 2006.

15. Lewis BP, Burge CB and Bartel DP: Conserved seed pairing, often flanked by adenosines, indicates that thousands of human genes are microRNA targets. Cell 120: 15-20, 2005.

16. Krek A, Grün D, Poy MN, et al: Combinatorial microRNA target predictions. Nat Genet 37: 495-500, 2005.

17. Volinia S, Calin GA, Liu CG, et al: A microRNA expression signature of human solid tumors defines cancer gene targets. Proc Natl Acad Sci USA 103: 2257-2261, 2006.

18. Massagué J: G1 cell-cycle control and cancer. Nature 432: 298-306, 2004.

19. Deshpande A, Sicinski P and Hinds PW: Cyclins and cdks in development and cancer: a perspective. Oncogene 24: 2909-2915, 2005.

20. Wang W, Zhao LJ, Tan YX, Ren H and Qi ZT: MiR-138 induces cell cycle arrest by targeting cyclin D3 in hepatocellular carcinoma. Carcinogenesis 33: 1113-1120, 2012.

21. Xu T, Zhu Y, Xiong Y, Ge YY, Yun JP and Zhuang SM: MicroRNA-195 suppresses tumorigenicity and regulates G1/S transition of human hepatocellular carcinoma cells. Hepatology 50: 113-121, 2009.

22. Baffa R, Fassan M, Volinia S, et al: MicroRNA expression profiling of human metastatic cancers identifies cancer gene targets. J Pathol 219: 214-221, 2009.

23. Gregory PA, Bert AG, Paterson EL, et al: The miR-200 family and miR-205 regulate epithelial to mesenchymal transition by targeting ZEB1 and SIP1. Nat Cell Biol 10: 593-601, 2008.

24. Ahn SM, Cha JY, Kim J, et al: Smad3 regulates E-cadherin via miRNA-200 pathway. Oncogene 31: 3051-3059, 2012.

25. Saydam O, Shen Y, Würdinger T, et al: Downregulated microRNA-200a in meningiomas promotes tumor growth by reducing E-cadherin and activating the Wnt/beta-catenin signaling pathway. Mol Cell Biol 29: 5923-5940, 2009.

26. Xia H, Ng SS, Jiang S, et al: miR-200a-mediated downregulation of ZEB2 and CTNNB1 differentially inhibits nasopharyngeal carcinoma cell growth, migration and invasion. Biochem Biophys Res Commun 391: 535-541, 2010.

27. Hung CS, Liu HH, Liu JJ, et al: MicroRNA-200a and -200b mediated hepatocellular carcinoma cell migration through the epithelial to mesenchymal transition markers. Ann Surg Oncol: Aug 7, 2012 (Epub ahead of print).

28. Lee JW, Park YA, Choi JJ, et al: The expression of the miRNA-200 family in endometrial endometrioid carcinoma. Gynecol Oncol 120: 56-62, 2011.

29. Grillo M, Bott MJ, Khandke $\mathrm{N}$, et al: Validation of cyclin D1/CDK4 as an anticancer drug target in MCF-7 breast cancer cells: effect of regulated overexpression of cyclin D1 and siRNA-mediated inhibition of endogenous cyclin D1 and CDK4 expression. Breast Cancer Res Treat 95: 185-194, 2006.

30. Bagchi A and Mills AA: The quest for the 1p36 tumor suppressor. Cancer Res 68: 2551-2556, 2008.

31. Sato Y, Kobayashi H, Suto Y, et al: Chromosomal instability in chromosome band 12p13: multiple breaks leading to complex rearrangements including cytogenetically undetectable sub-clones. Leukemia 15: 1193-1202, 2001. 